\title{
A Fault Diagnosis Method for Rolling Bearings Based on Feature Fusion of Multifractal Detrended Fluctuation Analysis and Alpha Stable Distribution
}

\author{
Qing Xiong, ${ }^{1}$ Weihua Zhang, ${ }^{1}$ Tianwei Lu, ${ }^{2}$ Guiming Mei, ${ }^{1}$ and Shulin Liang ${ }^{3}$ \\ ${ }^{1}$ State Key Laboratory of Traction Power, Southwest Jiaotong University, Chengdu, Sichuan 610031, China \\ ${ }^{2}$ School of Mechanical Engineering, Southwest Jiaotong University, Chengdu, Sichuan 610031, China \\ ${ }^{3}$ CNR Changchun Railway Vehicle Co., Changchun, Jilin 130062, China \\ Correspondence should be addressed to Qing Xiong; xiong_qiming@163.com
}

Received 28 April 2015; Accepted 31 August 2015

Academic Editor: Didier Rémond

Copyright (C) 2016 Qing Xiong et al. This is an open access article distributed under the Creative Commons Attribution License, which permits unrestricted use, distribution, and reproduction in any medium, provided the original work is properly cited.

\begin{abstract}
When rolling bearings fail, it is usually difficult to determine the degree of damage. To address this problem, a new fault diagnosis method was developed to perform feature extraction and intelligent classification of various fault position and damage degree of rolling bearing signals. Firstly, Multifractal Detrended Fluctuation Analysis (MFDFA) was used to compute five MFDFA features while five Alpha Stable Distribution (ASD) features were obtained by fitting the distribution to the vibration signals of each status and calculating the Probability Density Function (PDF). Secondly, Kernel Principle Component Analysis (KPCA) was used to achieve dimensionality reduction fusion of the combination of original features to gain the Kernel Principle Component Fusion Features (KPCFFs). Thirdly, the KPCFFs served as the input of Least Squares Support Vectors Machine (LSSVM) based on Particle Swarm Optimization (PSO) to assess rolling bearings' fault position and damage severity. Finally, the effectiveness of the method was validated by bench test data. The results demonstrated that the developed method can achieve intelligent diagnosis of rolling bearings' fault position and damage degree and can yield better diagnosis accuracy than single feature method or corresponding single feature fusion method.
\end{abstract}

\section{Introduction}

Rolling bearings are the key components of rotating machinery and their operational status directly influences the performance of the whole machine [1]. Critical work environment, such as high speed, heavy load, and repeated action of contact stress, makes it easy to deteriorate the operationalstatus of rolling bearings. The performance degradation of rolling bearings is a development process from minor faults to serious faults. In some cases, if rolling bearings appear as early minor faults, they do not need to be replaced. In order to effectively guide the maintenance of rolling bearings and save cost, the research on the defect severity of rolling bearings has caused wide attention. Bourdon et al. in [2] studied deeply the correlation between the length of the defect and Instantaneous Angular Speed (IAS) variations and then proposed a signal processing tool to reconstruct the IAS variations caused by the damage of rolling bearings. Finally, the effectiveness of the proposed tool is verified by different severities of spall defects. Ali et al. in [3], Zhang et al. in [4], and Kang et al. in [5], respectively, used empirical mode decomposition (EMD), ensemble empirical mode decomposition (EEMD), and wavelet transform (WT) to extract the fault features, revealed the relationship between entropy energy and defect severity, and then successfully distinguished the rolling bearings with different defect types and severities. Moreover, Rauber et al. in [6] and Sharma et al. in [7] considered the time-domain and frequency-domain indexes as the fault features, studied the changes of these indexes under various defect severities, and also obtained good results.

In addition to the above methods, the common fault feature extraction methods of rolling bearings include Wigner Ville distribution, spectral kurtosis, and envelope analysis. 
However, these methods tend to become unstable when used in complex signal processing [8-10]. Recently, Multifractal Detrended Fluctuation Analysis (MFDFA) method and Alpha Stable Distribution (ASD) method have been investigated to address this limitation. On one hand, there is a link between the multifractal spectrum parameters and the ASD parameters. Many groups have studied this connection and combined the advantages of both methods to make some achievements in human behavior analysis and signal modulation recognition [11-13]. On the other hand, MFDFA is able to characterize the internal dynamics mechanism of fault signal and to detect slight changes in complex environment of rotating machinery. The widely used ASD has good robustness in the modeling of pulse shape in non-Gauss signals. Therefore, both are very suitable for the fault diagnosis of rotating machinery, especially the rolling bearings [14-17]. Success combining of both methods may comprehensively utilize various information of fault features, reduce the uncertainty of diagnosis, and further improve the precision of diagnostic model.

The goal of this paper is to make further exploration on the fault feature extraction of rolling bearings with MFDFA and ASD and to achieve intelligent classification of different fault position and damage severity of rolling bearing signals.

\section{Basic Theory}

\subsection{MFDFA and MFDFA Features Extraction}

2.1.1. A Brief Introduction of MFDFA. The details about the theory and procedure of MFDFA were covered in [22, 23]. Its relationship to the classical multifractal theory is described below:

(1) If the mean value of $q$ th order fluctuation function $F_{q}(s)$ and the equal length $s$ have the relationship as follows:

$$
F_{q}(s) \sim s^{H(q)},
$$

the series is of multifractality, where $H(q)$ is the generalized Hurst exponent.

(2) The relationship between $H(q)$ and the scaling exponent $\tau(q)$ is as follows:

$$
\tau(q)=q H(q)-1
$$

(3) The relationship between the singularity exponent $h_{q}$ and the multifractal spectrum $f\left(h_{q}\right)$ is as follows:

$$
\begin{gathered}
h_{q}=\tau^{\prime}(q)=H(q)+q H^{\prime}(q), \\
f\left(h_{q}\right)=q h_{q}-\tau(q)=q\left[h_{q}-H(q)\right]+1 .
\end{gathered}
$$

2.1.2. MFDFA Features Extraction. The multifractal spectrum calculated by MFDFA is a parameter set that can fully describe the dynamics behavior of multifractal time series. The failure of rolling bearings will lead to changes in the parameters of multifractal spectrum. This paper adopts five common multifractal spectrum parameters as the MFDFA features (as shown in Figure 1).

The spectral width $\Delta h_{q}=h_{q \max }-h_{q \text { min }}$ reflects the unevenness of probability distribution of the fractal structure. The larger the $\Delta h_{q}$, the greater the unevenness degree and the stronger the multifractality.

The singularity exponent corresponding to the pole $h_{q 0}\left(f_{\max }=f\left(h_{q 0}\right), h_{q 0} \in\left[h_{q \min }, h_{q \max }\right]\right)$ reflects the randomness of the vibration signal. The larger the $h_{q 0}$, the more irregular the vibration signal and the stronger the randomness.

The fractal dimension difference of probability subset $\Delta f=f\left(h_{q \max }\right)-f\left(h_{q \min }\right)$ reflects the proportion of small peak and large peak of the vibration signal. If $\Delta f<0$, the maximum probability subset number is greater than the minimum probability subset number, and vice versa.

The left endpoint $h_{q \text { min }}$ and the right endpoint $h_{q \max }$ represent the maximum and minimum fluctuation of singularity exponent, respectively.

\subsection{ASD and ASD Features Extraction}

2.2.1. A Brief Introduction of ASD. ASD was developed from the generalized central limit theorem. It has more extensive applicability than the Gaussian distribution. Gaussian distribution, Cauchy distribution, and Levy distribution are three special cases of it. The theory and procedure of ASD can be found in document $[24,25]$.

ASD does not have a closed expression of PDF. Hence generally characteristic function is used to describe its statistical properties. Its characteristic function can be expressed as

$$
\varphi(u)=\exp \left\{j \delta u-\gamma|u|^{\alpha}[1+j \beta \operatorname{sgn}(u) \omega(u, \alpha)]\right\},
$$

where

$$
\begin{aligned}
& \omega(u, \alpha)= \begin{cases}\tan \left(\frac{\pi \alpha}{2}\right), & \alpha \neq 1 \\
\left(\frac{2}{\pi}\right) \log |u|, & \alpha=1,\end{cases} \\
& \operatorname{sgn}(u)= \begin{cases}1, & u>0 \\
0, & u=0 \\
-1, & u<0 .\end{cases}
\end{aligned}
$$

2.2.2. ASD Features Extraction. As (4) shows, the statistical characteristics of ASD are completely determined by four parameters $(\alpha, \beta, \gamma, \delta)$. In [16], it has been proved that the use of the four parameters of ASD as the fault features in fault diagnosis of rolling bearings is operable. In addition, the author found that if a rolling bearing fault occurs, not only will the above four parameters change, but also the extremum of PDF $h$ has a clear variation. Therefore, this paper will use the following five parameters as ASD features (as shown in Figure 1).

The characteristic exponent $\alpha(0<\alpha \leq 2)$ reflects the tail thickness of ASD. The smaller the $\alpha$, the thicker the tail, and the stronger the signal pulse. 

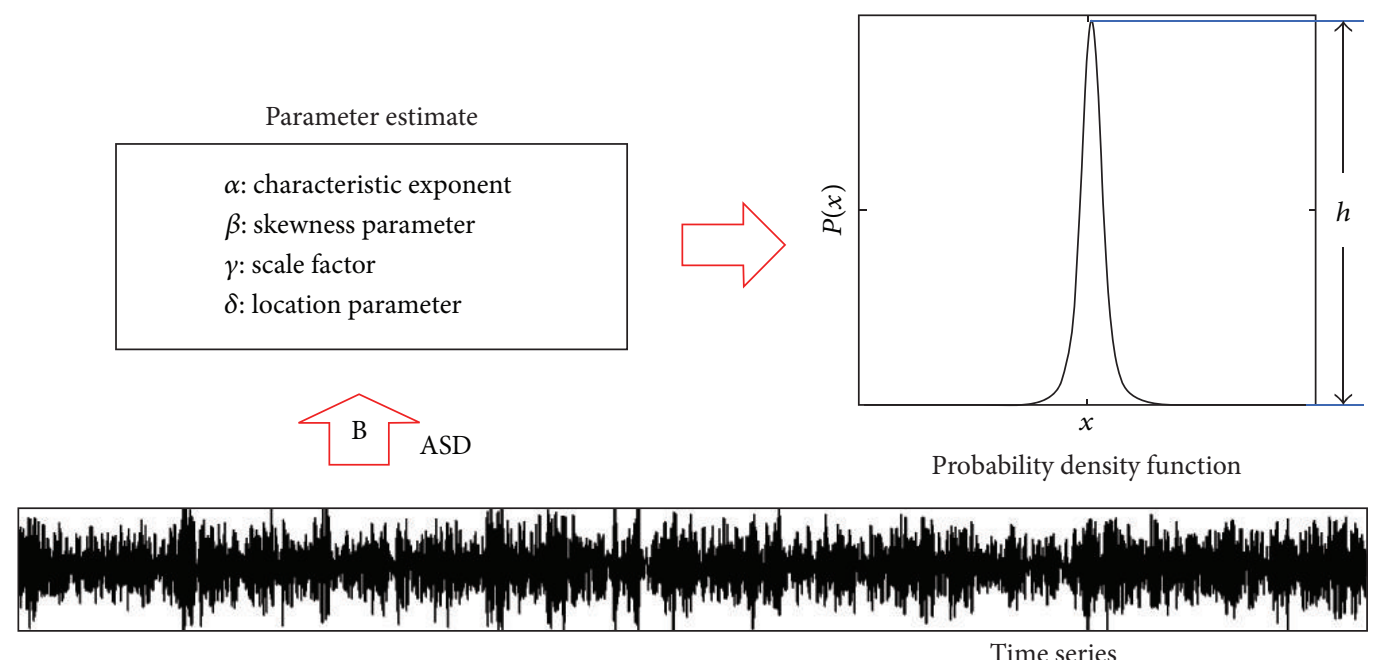

A $\angle$ MFDFA

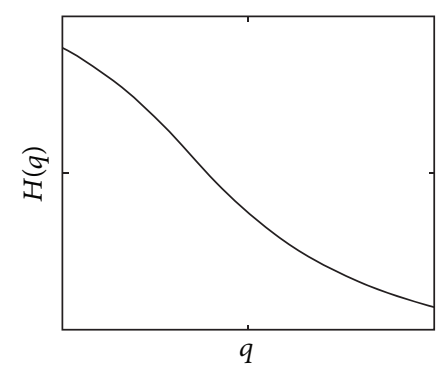

Hurst exponent

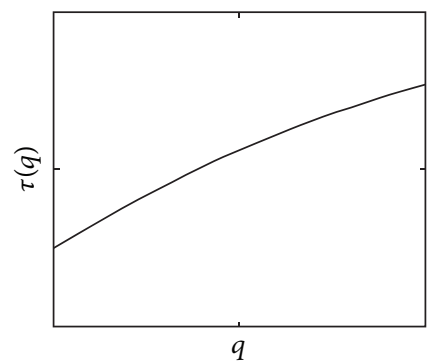

Scaling exponent

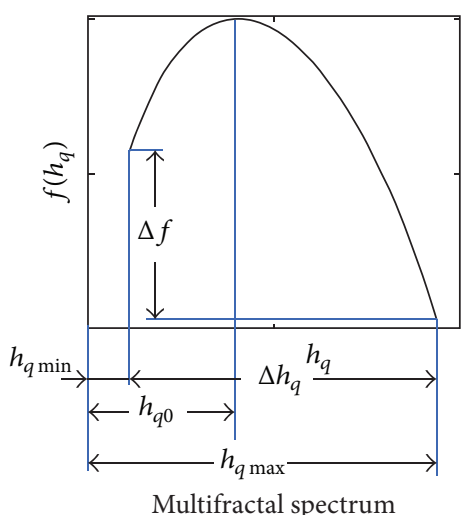

FIgURE 1: Schematic diagram of MFDFA features and ASD features.

The skewness parameter $\beta(-1 \leq \beta \leq 1)$ represents the skewness of ASD. When $\beta=0$, the PDF is symmetric about the location parameter $\delta(-\infty \leq \delta \leq \infty)$. of ASD.

The scale factor $\gamma(\gamma>0)$ determines the scattered degree

The extremum of PDF $h(h>0)$ equals the probability when transient amplitude is zero. The smaller the $h$, the greater the probability density of large amplitude of vibration signals, and the more scattered the curve.

\subsection{Other Theories}

2.3.1. Brief Introduction of KPCA, PSO, and LSSVM. Kernel Principle Component Analysis (KPCA) is the nonlinear generalization of principle component analysis (PCA). The data samples of input space are mapped to a high-dimensional feature space by nonlinear mapping; then the nonlinear structure information of data samples can be extracted by PCA. The procedure of KPCA can be found in $[26,27]$.

Particle Swarm Optimization (PSO) originated in the study of birds feeding behavior, which is a kind of widely used evolutionary computation theory. The theory and procedure of PSO were covered in [28]. The update equation is

$$
\begin{aligned}
& X^{t+1}=X^{t}+V^{t+1}, \\
& V^{t+1}=V^{t}+c_{1} r_{1}\left(P_{i d}^{t}-x^{t}\right)+c_{2} r_{2}\left(P_{g d}^{t}-x^{t}\right),
\end{aligned}
$$

where $t$ is iteration number. $X^{t}$ and $V^{t}$ are, respectively, the position and velocity of particles. $r_{1}$ and $r_{2}$ are two independent random numbers in the range of $[0,1] \cdot c_{1}$ and $c_{2}$ are two positive constants named acceleration coefficients. $P_{i d}$ and $P_{g d}$ are, respectively, the local best and global best.

The algorithm of Least Squares Support Vectors Machine (LSSVM) proposed by Suykens and Vandewalle [29] is a generalization of the support vectors machine (SVM). It is also a kind of regression prediction algorithm but is more efficient than SVM. The selection of kernel function is the key of LSSVM method. The common kernel function includes radial basis function (RBF), polynomial kernel function, and sigmoid kernel function. Because RBF requires the least 


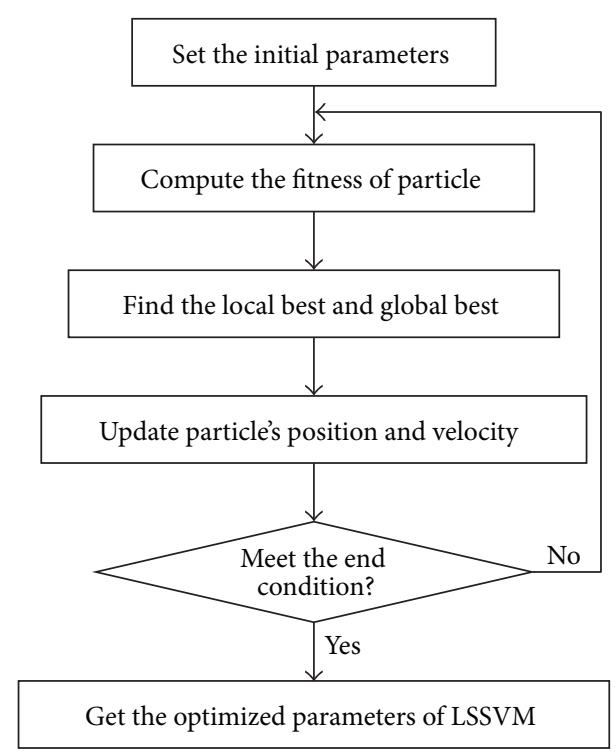

FIGURE 2: Process of optimizing the LSSVM parameters with PSO.

parameters (only the regularization parameter $\lambda$ and kernel parameter $\sigma$ ), we select it to construct the LSSVM.

\subsubsection{The Parameter Optimization Method of LSSVM Based} on PSO. Parameters $\lambda$ and $\sigma$ have a great impact on the classification accuracy of LSSVM. At present, besides the PSO, the optimized methods of the two parameters mainly include cross validation method and modeling experience statistics method [30].

Cross validation method is difficult to determine a reasonable parameter range in advance to a certain extent and hence will affect the speed and accuracy of fault diagnosis. Modeling experience statistics method requires the accumulation of long-term experiment. Therefore, this paper uses PSO to optimize the two parameters of LSSVM. Figure 2 is the parameter optimization process.

The specific steps are illustrated as follows:

(1) Initialization settings are as follows: set population size, iteration number, initial position and velocity, and so forth.

(2) Compute the particle fitness value: using LSSVM corresponding to each particle vector to predict the training samples. The prediction error value of the current position of the particle is used as the fitness value.

(3) Find the optimal particle location: comparing current fitness value with the best fitness value of the particle and updating the optimal position of the particle based on the fitness value.

(4) Find the optimal population location: comparing the best fitness value of the particle with the best fitness value of the population and updating the optimal position of the population based on the fitness value.

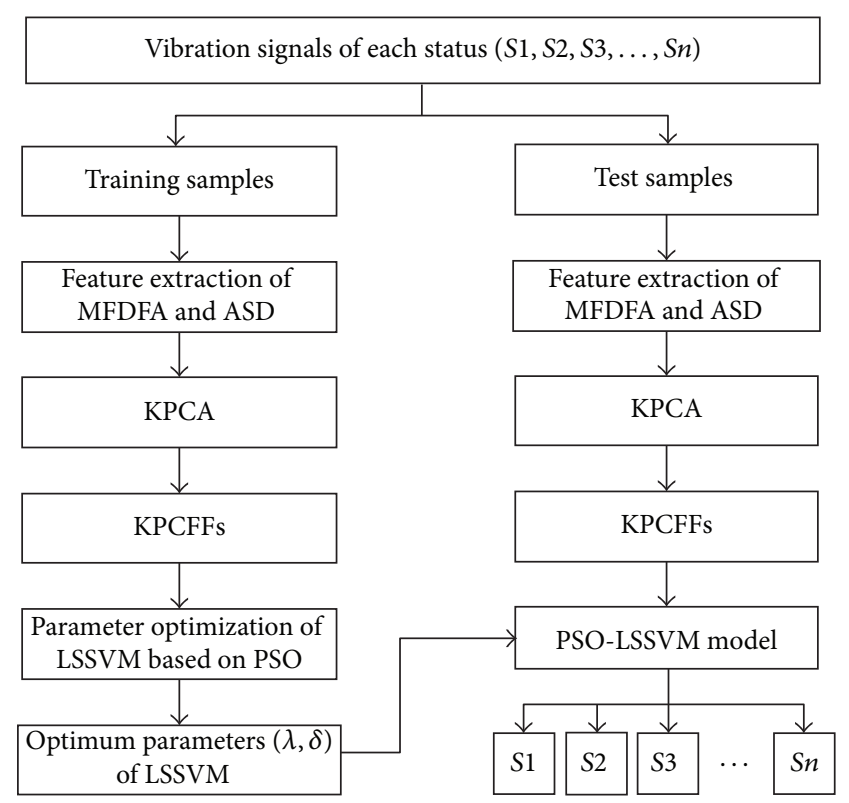

FIGURE 3: The diagnosis model based on feature fusion of MFDFA and ASD.

(5) Update the position and velocity of particles: iterative calculation according to formula (6).

(6) Check the end condition; if not met, return to step (2) to the further iterative calculation until meeting the end conditions, and then output the results.

\section{A Fault Diagnosis Method for Rolling Bearings Based on Feature Fusion of MFDFA and ASD}

MFDFA features and ASD features contain the fault information of rolling bearings in different status space. Fuse the two kinds of features and heterogeneous information can complement each other. More abundant information than single signal feature can be obtained.

Combining the two features in a serial manner is the simplest feature fusion method, but the high dimensionality and redundancy of combined features easily result in the decline of classifier's recognition ability. KPCA has a strong capacity of dimension reduction fusion and redundant elimination. Use KPCA on the combined features; the KPCFFs which are beneficial for fault classification can be easily obtained.

Finally, PSO-LSSVM was applied to the KPCFFs to identify the status of rolling bearings. The diagnosis model constructed in this paper is shown in Figure 3.

The specific process is described as follows:

(1) Obtain $n$ status of vibration signals of rolling bearings, including normal, inner-race faults (different damage degree), outer-race faults (different damage degree), and ball faults (different damage degree), labeled as $S 1, S 2, S 3, \ldots, S n$, respectively. 
TABLE 1: The detailed descriptions of seven vibration signals.

\begin{tabular}{lcccc}
\hline Data label & Operational status & Fault size $(\mathrm{mm})$ & Speed $(\mathrm{r} / \mathrm{min})$ & Sampling frequency $(\mathrm{kHZ})$ \\
\hline S1 & Normal & 0 & 1797 & 12 \\
S2 & Slight inner-race faults & 0.18 & 1797 & 12 \\
S3 & Serious inner-race faults & 0.54 & 1797 & 12 \\
S4 & Slight outer-race faults & 0.18 & 1797 & 12 \\
S5 & Serious outer-race faults & 0.54 & 1797 & 12 \\
S6 & Slight ball faults & 0.18 & 1797 & 12 \\
S7 & Serious ball faults & 0.54 & 1797 & 12 \\
\hline
\end{tabular}

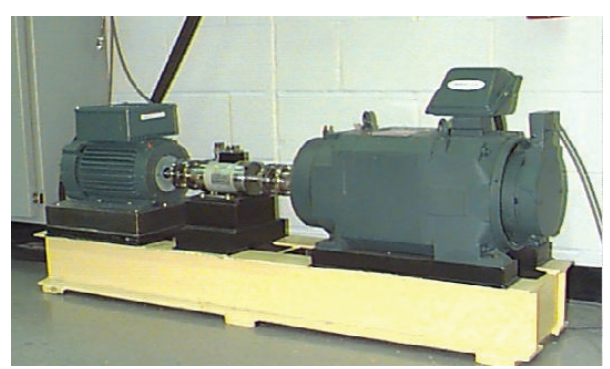

(a) Experimental setup

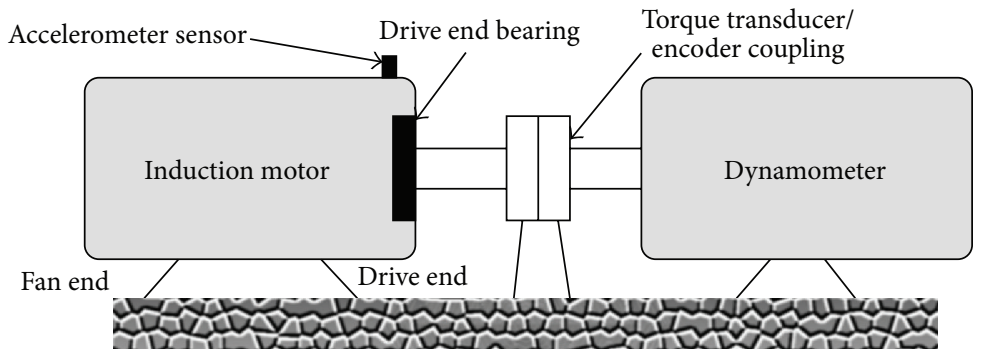

(b) Schematic diagram of the experimental setup

Figure 4: Experimental system.

(2) Divide the vibration signal of each status into training samples and test samples.

(3) Extract MFDFA features and ASD features of training samples; combine the ten features in a serial manner to get a combined feature set.

(4) Use KPCA on the combined features to obtain the KPCFFs.

(5) Consider the fusion feature as input samples; optimize two parameters of LSSVM by PSO. Obtain the optimal parameters $\lambda$ and $\sigma$; then establish the PSOLSSVM model.

(6) Take the same processing on the test samples, put the KPCFFs of test samples into the PSO-LSSVM model which was well trained, and determine the working condition and fault type of the roller bearings according to the output of the classifier.

\section{Applications}

4.1. Data Source. The data of rolling bearing is obtained from the Bearing Data Center of Case Western Reserve University (CWRU) [31]. The data is acquired by the bench test after setting some artificial faults. The type of test bearing is deep groove ball bearing (SKF 6205-2RS JEM). Figure 4 shows the experimental system.

It can be seen from Figure 4 that the rolling bearing is installed in the mechanical system driven by a motor; the vibration acceleration sensor is vertically fixed on the shell at the top of drive end bearing; the types of collected vibration signal of rolling bearing include normal, inner-race faults, outer-race faults, and the ball faults. In this paper, seven kinds of status data will be selected (sample length of each status is 120000) to verify the effectiveness of the proposed method. The detailed descriptions of seven vibration signals are shown in Table 1.

The waveforms of seven vibration signals are shown in Figure 5. The figure shows that only from the time-domain signal is it very difficult to distinguish the seven statuses of the bearing.

4.2. MFDFA Features Extraction. Dividing evenly each status data item into twenty segments, the length of each segment is 6000 . Ten segments were randomly selected (as the training samples) from the twenty segments to perform MFDFA analysis. After averaging, the multifractal spectrums shown in Figure 6 can be obtained. Five MFDFA features were extracted from each signal in all seven kinds of signals, as shown in Table 2 (due to space limitations, only the top five sets of data were listed here).

It can be seen from Figure 6 that the size, shape, and location of multifractal spectrums of the vibration signal in each status are different. Also obvious difference exists in five parameters of multifractal spectrums (Table 2). It shows that the seven signals have different intrinsic dynamic mechanism and multifractal properties.

Table 2 shows, under different statuses, the values of five MFDFA features of each status are not identical. The values of 


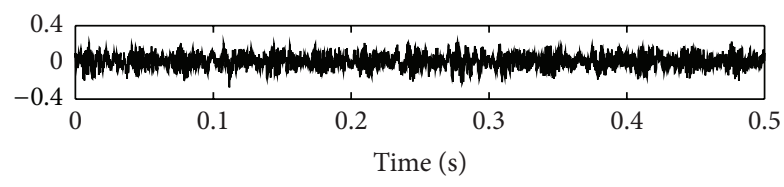

(a) Normal

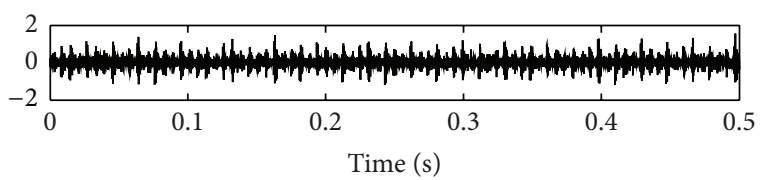

(b) Slight inner-race faults

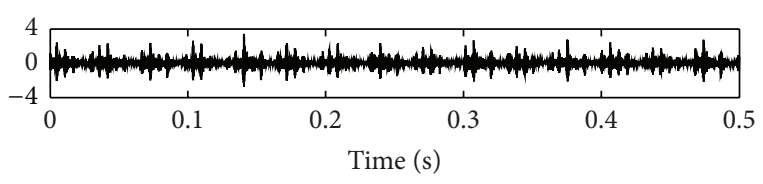

(c) Serious inner-race faults

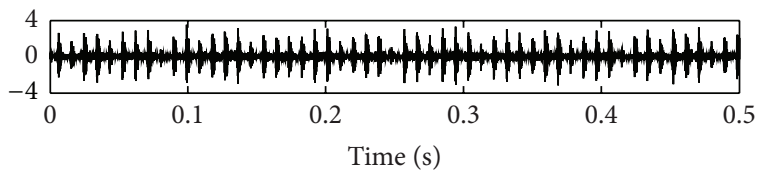

(d) Slight outer-race faults

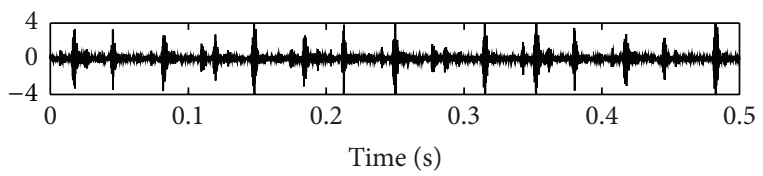

(e) Serious outer-race faults

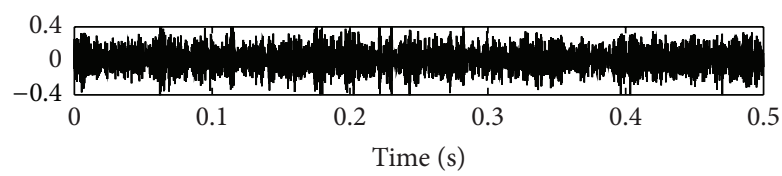

(f) Slight ball faults

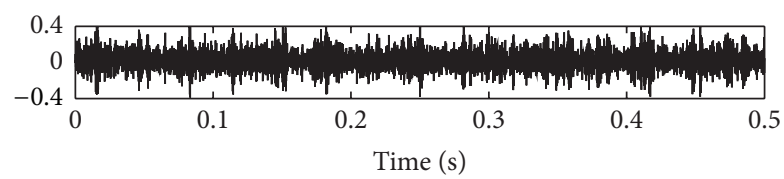

(g) Serious ball faults

FIGURE 5: The waveforms of seven vibration signals (ordinate unit: $\left.\mathrm{m} / \mathrm{s}^{2}\right)$.

$h_{q 0}$ or $h_{q \min }$ are not only able to distinguish whether there is a fault in the rolling bearing (normal $\gg$ failure), but also can describe the fault degree under the same fault position (slight faults $>$ serious faults).

The values of $h_{q \max }$ are not only able to distinguish whether there is a fault in the rolling bearing (normal $\gg$ failure), but also can describe the fault degree under the same fault position (slight faults < serious faults).

4.3. ASD Features Extraction. We estimated the parameters of ASD by using the empirical characteristic function method [32] and calculated the PDFs of the above ten segments data.

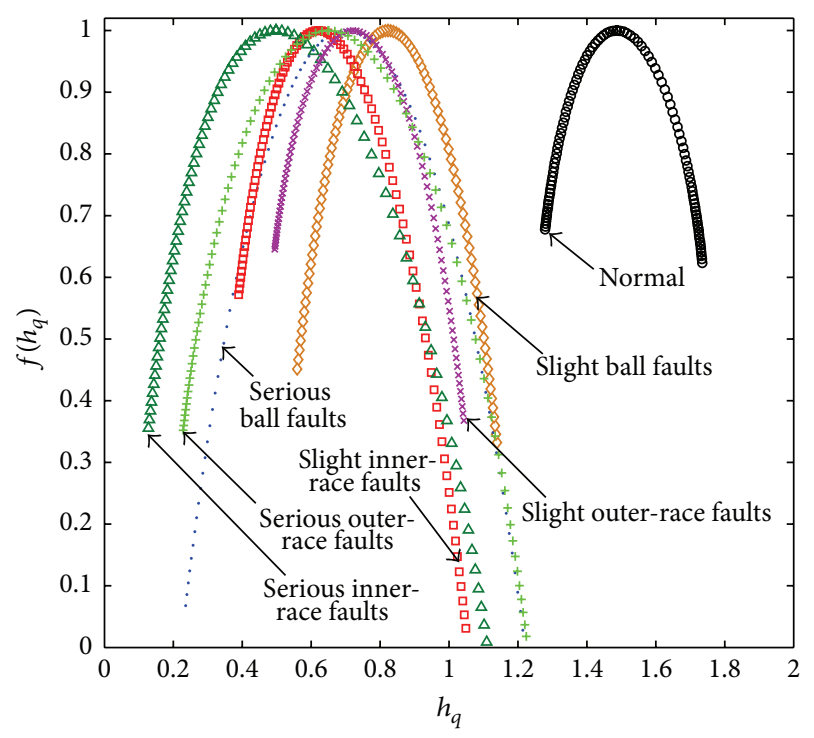

Figure 6: The multifractal spectrum.

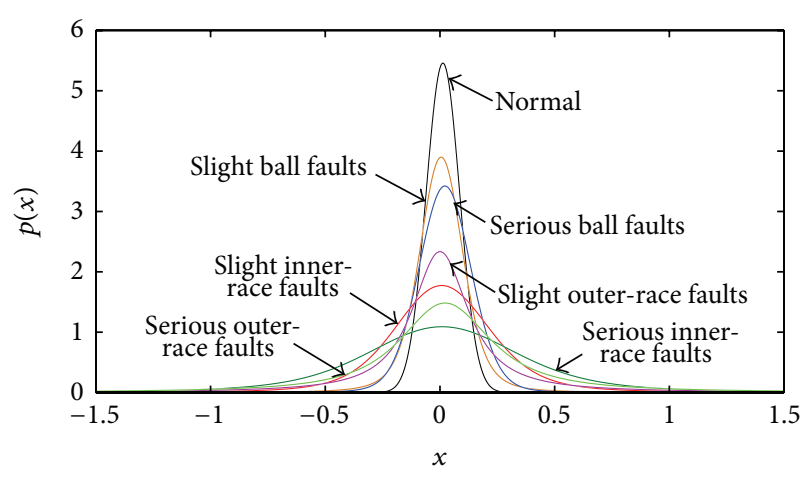

Figure 7: Probability Density Function.

After averaging, the PDFs of each status as shown in Figure 7 can be obtained. We extracted five ASD features from seven kinds of signals, such as shown in Table 3 (similarly, only the top five sets of data were listed here).

It can be seen from Figure 7 that the size, shape, and location of PDFs of the vibration signal in each status are different, so is the obvious difference in the extremum of PDFs $h$. It shows that the seven signals have different statistical properties.

Table 3 shows, under different statuses, that the values of the five ASD features of each status are not identical. The normal signal, $\alpha=2$, can be considered to be subject to Gaussian normal distribution; the fault signals, $\alpha<2$, are non-Gaussian signals.

The values of $\alpha$ are not only able to distinguish whether there is a fault in the rolling bearing (normal $>$ ball faults $>$ inner-race faults $>$ outer-race faults), but also can describe the fault degree under the same fault position (slight faults $>$ serious faults ).

The values of $h$ are not only able to distinguish whether there is a fault in the rolling bearing (normal $\gg$ failure), 
TABLE 2: The MFDFA features of seven vibration signals.

\begin{tabular}{|c|c|c|c|c|c|}
\hline Operational status & $h_{q 0}$ & $h_{q \min }$ & $h_{q \max }$ & $\Delta h_{q}$ & $\Delta f$ \\
\hline \multirow{5}{*}{ Normal (S1) } & 1.4725 & 1.2808 & 1.8472 & 0.5664 & 0.3800 \\
\hline & 1.4722 & 1.2654 & 1.8168 & 0.5514 & 0.2748 \\
\hline & 1.4877 & 1.2772 & 1.8705 & 0.5933 & 0.3545 \\
\hline & 1.4799 & 1.2853 & 1.8272 & 0.5419 & 0.2783 \\
\hline & 1.4800 & 1.2574 & 1.8204 & 0.5629 & 0.2135 \\
\hline \multirow{5}{*}{ Slight inner-race faults $(S 2)$} & 0.6210 & 0.3895 & 1.0487 & 0.6592 & 0.5408 \\
\hline & 0.6206 & 0.3845 & 1.0313 & 0.6468 & 0.5008 \\
\hline & 0.6211 & 0.3975 & 1.0288 & 0.63145 & 0.5117 \\
\hline & 0.6258 & 0.3873 & 1.0008 & 0.6136 & 0.3667 \\
\hline & 0.6169 & 0.3924 & 1.0073 & 0.6149 & 0.4744 \\
\hline \multirow{5}{*}{ Serious inner-race faults $(S 3)$} & 0.4979 & 0.1277 & 1.1092 & 0.9815 & 0.3466 \\
\hline & 0.5089 & 0.1648 & 1.1146 & 0.9498 & 0.4304 \\
\hline & 0.4974 & 0.1403 & 1.1050 & 0.9648 & 0.3707 \\
\hline & 0.5001 & 0.1439 & 1.0918 & 0.9479 & 0.3793 \\
\hline & 0.5020 & 0.1406 & 1.1218 & 0.9812 & 0.3815 \\
\hline \multirow{5}{*}{ Slight outer-race faults (S4) } & 0.7213 & 0.4941 & 1.0430 & 0.5488 & 0.2776 \\
\hline & 0.7266 & 0.5327 & 1.0283 & 0.4956 & 0.3233 \\
\hline & 0.7145 & 0.5035 & 1.0283 & 0.5249 & 0.3175 \\
\hline & 0.7342 & 0.5209 & 1.0896 & 0.5688 & 0.4295 \\
\hline & 0.7380 & 0.5308 & 1.0284 & 0.4976 & 0.2593 \\
\hline \multirow{5}{*}{ Serious outer-race faults (S5) } & 0.6497 & 0.2285 & 1.2242 & 0.9957 & 0.3342 \\
\hline & 0.6627 & 0.2446 & 1.1853 & 0.9407 & 0.2665 \\
\hline & 0.6344 & 0.2275 & 1.1913 & 0.9638 & 0.3470 \\
\hline & 0.6567 & 0.2769 & 1.2264 & 0.9494 & 0.4663 \\
\hline & 0.6442 & 0.2652 & 1.1654 & 0.9002 & 0.3383 \\
\hline \multirow{5}{*}{ Slight ball faults (S6) } & 0.8228 & 0.5595 & 1.1391 & 0.5796 & 0.1190 \\
\hline & 0.8236 & 0.5575 & 1.1098 & 0.5523 & 0.0146 \\
\hline & 0.8184 & 0.5595 & 1.1428 & 0.5833 & 0.1309 \\
\hline & 0.8178 & 0.5507 & 1.0857 & 0.5350 & -0.0412 \\
\hline & 0.8225 & 0.5657 & 1.1536 & 0.5879 & 0.1741 \\
\hline \multirow{5}{*}{ Serious ball faults (S7) } & 0.6992 & 0.2357 & 1.2144 & 0.9787 & 0.0401 \\
\hline & 0.6995 & 0.2265 & 1.2210 & 0.9945 & 0.0243 \\
\hline & 0.6966 & 0.2496 & 1.2081 & 0.9584 & 0.0908 \\
\hline & 0.6901 & 0.2328 & 1.2155 & 0.9827 & 0.0868 \\
\hline & 0.6978 & 0.2295 & 1.2201 & 0.9906 & 0.0592 \\
\hline
\end{tabular}

but also can describe the fault degree under the same fault position (slight faults $>$ serious faults ).

Except for the normal signal, the value of $\beta$ of all the rest signals is close to zero, indicating that not only is the PDF of normal signal symmetric, but also all the PDFs of fault signals are almost symmetric. This is because of the actual symmetric structure of the bearing.

4.4. Feature Fusion. By combining the MFDFA features and ASD features of the training samples in a serial manner, a feature matrix with size of $70 \times 10$ can be obtained. 70 lines represent all samples of seven statuses signals; 10 columns represent the feature vector of each sample. We used KPCA to fuse the combined features and selected kernel principal component according to the cumulative contribution rate higher than or equal to 95\%; a KPCFF matrix with size of $70 \times 5$ can be obtained, as shown in Table 4 (similarly, only the top five sets of fusion feature were listed here).

If we choose the first and second KPCFFs to be abscissa and ordinate, respectively, the result of classification of seven vibration signals can be obtained as shown in Figure 8. It can be seen from Figure 8 that the training samples are well classified only relying on the first two KPCFFs, and the seven vibration signals dispersed in seven distinct areas, which further demonstrates the correctness and effectiveness of the proposed method in this paper. 
TABLE 3: The ASD features of seven vibration signals.

\begin{tabular}{|c|c|c|c|c|c|}
\hline Operational status & $\alpha$ & $\beta$ & $\gamma$ & $\delta$ & $h$ \\
\hline \multirow{5}{*}{ Normal (S1) } & 2.0000 & -1.0000 & 0.0530 & 0.0120 & 5.4785 \\
\hline & 2.0000 & -1.0000 & 0.0513 & 0.0104 & 5.5043 \\
\hline & 2.0000 & -1.0000 & 0.0517 & 0.0113 & 5.3190 \\
\hline & 2.0000 & -1.0000 & 0.0527 & 0.0119 & 5.4111 \\
\hline & 2.0000 & -1.0000 & 0.0528 & 0.0097 & 5.4430 \\
\hline \multirow{5}{*}{ Slight inner-race faults (S2) } & 1.6510 & 0.0351 & 0.1676 & 0.0147 & 1.7729 \\
\hline & 1.6462 & 0.0431 & 0.1667 & 0.0149 & 1.7670 \\
\hline & 1.6439 & 0.0443 & 0.1663 & 0.0152 & 1.7762 \\
\hline & 1.6412 & 0.0478 & 0.1659 & 0.0154 & 1.7890 \\
\hline & 1.6482 & 0.0368 & 0.1681 & 0.0146 & 1.8081 \\
\hline \multirow{5}{*}{ Serious inner-race faults (S3) } & 1.4628 & -0.0055 & 0.2322 & 0.0113 & 1.1271 \\
\hline & 1.4615 & -0.0388 & 0.2323 & 0.0057 & 1.2140 \\
\hline & 1.4572 & -0.0244 & 0.2306 & 0.0081 & 1.0944 \\
\hline & 1.4638 & -0.0169 & 0.2342 & 0.0098 & 1.1112 \\
\hline & 1.4571 & -0.0134 & 0.2288 & 0.0102 & 1.0653 \\
\hline \multirow{5}{*}{ Slight outer-race faults $(S 4)$} & 1.1366 & 0.0144 & 0.1991 & 0.0319 & 2.3344 \\
\hline & 1.1338 & 0.0024 & 0.1974 & 0.0215 & 2.3226 \\
\hline & 1.1315 & 0.0232 & 0.1977 & 0.0384 & 2.3404 \\
\hline & 1.1277 & 0.0210 & 0.1962 & 0.0371 & 2.2661 \\
\hline & 1.1432 & 0.0368 & 0.1881 & 0.0146 & 2.2963 \\
\hline \multirow{5}{*}{ Serious outer-race faults (S5) } & 1.0429 & 0.0300 & 0.1293 & 0.0586 & 1.4839 \\
\hline & 1.0406 & 0.0427 & 0.1287 & 0.0864 & 1.4733 \\
\hline & 1.0482 & 0.0313 & 0.1292 & 0.0544 & 1.4918 \\
\hline & 1.0447 & 0.0265 & 0.1299 & 0.0498 & 1.4810 \\
\hline & 1.0446 & 0.0394 & 0.1296 & 0.0735 & 1.4785 \\
\hline \multirow{5}{*}{ Slight ball faults (S6) } & 1.9577 & 0.3325 & 0.0784 & 0.0225 & 3.8988 \\
\hline & 1.9572 & 0.2264 & 0.0788 & 0.0209 & 3.8121 \\
\hline & 1.9528 & 0.3042 & 0.0785 & 0.0197 & 3.8065 \\
\hline & 1.9452 & 0.3021 & 0.0783 & 0.0182 & 3.9182 \\
\hline & 1.9612 & 0.3226 & 0.0788 & 0.0217 & 3.8847 \\
\hline \multirow{5}{*}{ Serious ball faults (S7) } & 1.7828 & 0.0582 & 1.3278 & 0.0125 & 3.4243 \\
\hline & 1.7746 & 0.0608 & 1.3192 & 0.0034 & 3.4013 \\
\hline & 1.7843 & 0.0667 & 1.3177 & -0.0043 & 3.4117 \\
\hline & 1.7920 & 0.0674 & 1.3194 & -0.0094 & 3.4339 \\
\hline & 1.7896 & 0.0644 & 1.3081 & -0.0113 & 3.4218 \\
\hline
\end{tabular}

4.5. Intelligent Diagnosis. We then put the KPCFFs obtained in Section 4.4 into the PSO-LSSVM classifier for training. The initial parameters of PSO are as follows: population size is 40; the maximum iteration is 200; the initial position and initial velocity are generated randomly; acceleration coefficients $c_{1}$ and $c_{2}$ are both 2; optimal ranges of $\lambda$ and $\sigma$ are $0.1 \sim 100$ and $0.01 \sim 1000$, respectively. After the iteration, we obtained the optimal parameters for LSSVM: $\lambda=0.1$ and $\sigma=0.67895$.

At the same time, we used the remaining ten segments data as the test samples to extract the MFDFA features and ASD features, then got the five-dimensional KPCFFs through KPCA, and finally put it into the PSO-LSSVM model which was well trained to classify. In order to reduce the amount of calculation, we chose the minimum output coding (MOC) as the coding method. For seven different statuses, adopting three classifiers can meet the requirement of diagnosis. The specific coding is as follows:

$$
c_{b}=\left[\begin{array}{ccccccc}
-1 & -1 & -1 & -1 & 1 & 1 & 1 \\
-1 & -1 & 1 & 1 & -1 & -1 & 1 \\
-1 & 1 & -1 & 1 & -1 & 1 & -1
\end{array}\right]
$$

In the formula above, column 1 to column 7, respectively, correspond to seven statuses of rolling bearing $(S 1 \sim S 7)$. In order to clearly show the advantages of this feature fusion method in the fault diagnosis of rolling bearing, we also feed the original MFDFA features, original ASD features, MFDFA features with KPCA, and ASD features with KPCA into the same PSO-LSSVM model for the comparison of classification 
TABle 4: Kernel Principle Component Fusion Features.

\begin{tabular}{|c|c|c|c|c|c|}
\hline Operational status & The first KPCFF & The second KPCFF & The third KPCFF & The fourth KPCFF & The fifth KPCFF \\
\hline \multirow{5}{*}{ Normal (S1) } & 4.9573 & -1.0961 & -1.2839 & 0.9007 & -0.1812 \\
\hline & 5.0354 & -0.8159 & -1.0046 & 0.5238 & -0.0966 \\
\hline & 4.9983 & -0.9952 & -1.3666 & 0.7806 & -0.2376 \\
\hline & 5.0838 & -0.8831 & -1.0041 & 0.5002 & -0.0809 \\
\hline & 5.0996 & -0.6343 & -0.9436 & 0.2673 & -0.0982 \\
\hline \multirow{5}{*}{ Slight inner-race faults (S2) } & -1.1187 & -0.7731 & 0.2512 & 1.6153 & -0.2885 \\
\hline & -1.1032 & -0.6949 & 0.3918 & 1.4490 & -0.2378 \\
\hline & -1.0877 & -0.7556 & 0.4253 & 1.5020 & -0.1996 \\
\hline & -0.9460 & -0.4249 & 0.7763 & 0.8910 & -0.1002 \\
\hline & -1.0461 & -0.6578 & 0.5738 & 1.3861 & -0.1424 \\
\hline \multirow{5}{*}{ Serious inner-race faults (S3) } & -1.9727 & 0.2010 & -0.7469 & 0.5437 & -0.6306 \\
\hline & -1.9041 & 0.0533 & -0.8268 & 1.1150 & -0.5201 \\
\hline & -1.9308 & 0.1812 & -0.7419 & 0.7634 & -0.5559 \\
\hline & -1.9250 & 0.1193 & -0.6717 & 0.7631 & -0.5341 \\
\hline & -1.9555 & 0.1244 & -0.8327 & 0.7224 & -0.6227 \\
\hline \multirow{5}{*}{ Slight outer-race faults (S4) } & -0.7860 & -1.1351 & 0.6127 & -0.3092 & 1.0381 \\
\hline & -0.6851 & -1.1030 & 0.7347 & 0.2631 & 1.2416 \\
\hline & -0.8711 & -1.4199 & 0.6346 & -0.3111 & 1.0466 \\
\hline & -0.9288 & -1.6412 & 0.1951 & 0.1508 & 0.9099 \\
\hline & -0.5976 & -1.7833 & 0.9016 & 0.1909 & 1.2657 \\
\hline \multirow{5}{*}{ Serious outer-race faults (S5) } & -1.9878 & -1.4426 & -1.2981 & -1.3160 & -0.2770 \\
\hline & -2.0303 & -2.0079 & -1.0134 & -2.3885 & -0.2986 \\
\hline & -2.0095 & -1.4012 & -1.1461 & -1.0860 & -0.1815 \\
\hline & -1.9680 & -1.6459 & -1.3654 & -0.4349 & -0.1411 \\
\hline & -2.0229 & -1.9337 & -0.9398 & -1.6368 & -0.1383 \\
\hline \multirow{5}{*}{ Slight ball faults (S6) } & 0.6667 & 0.2965 & 1.7823 & -0.5872 & -0.7083 \\
\hline & 0.8826 & 0.5191 & 1.9752 & -0.9107 & -0.5503 \\
\hline & 0.6924 & 0.3294 & 1.7192 & -0.4468 & -0.6831 \\
\hline & 0.8585 & 0.7214 & 2.2533 & -1.0845 & -0.4724 \\
\hline & 0.6424 & 0.1901 & 1.6329 & -0.3335 & -0.7461 \\
\hline \multirow{5}{*}{ Serious ball faults (S7) } & -0.3411 & 2.8973 & -0.5789 & -1.1231 & 0.2425 \\
\hline & -0.2971 & 3.1559 & -0.5847 & -0.9404 & 0.2741 \\
\hline & -0.2901 & 3.1244 & -0.5308 & -0.3762 & 0.3756 \\
\hline & -0.2722 & 3.3138 & -0.5837 & -0.2720 & 0.3358 \\
\hline & -0.2391 & 3.4092 & -0.5689 & -0.3363 & 0.3301 \\
\hline
\end{tabular}

results. After decoding, the diagnosis results with various methods are shown in Table 5.

It can be seen from Table 5, under the condition of training samples and test samples which are both 70, that five methods based on MFDFA features or ASD features can effectively distinguish the status of the test samples. This once again shows that the vibration signals of rolling bearing have multifractal properties and obey the Alpha Stable Distribution and that their parameters can be used as the fault features to describe the variation trend of operational status and to distinguish different fault position and fault degree of rolling bearing.

Regarding the generalization ability, the feature fusion method based on MFDFA features and ASD features has only one mistake (a sample originally belongs to $S 4$ but was classified as another category); the diagnostic accuracy is $98.6 \%$, significantly higher than the single feature method and the corresponding single feature fusion method. It demonstrated that, compared with the single feature method, KPCFFs make full use of the redundant and complementary information of heterogeneous source and the nonlinear relation between the original features, gain more comprehensive information in the operational status of rolling bearing, enhance the recognition ability of the classifier, and hence improve the accuracy of the diagnosis.

4.6. Discussion and Comparison with Some Previous Works. To further show the effectiveness of the proposed method, a comparison between the present work and the works 


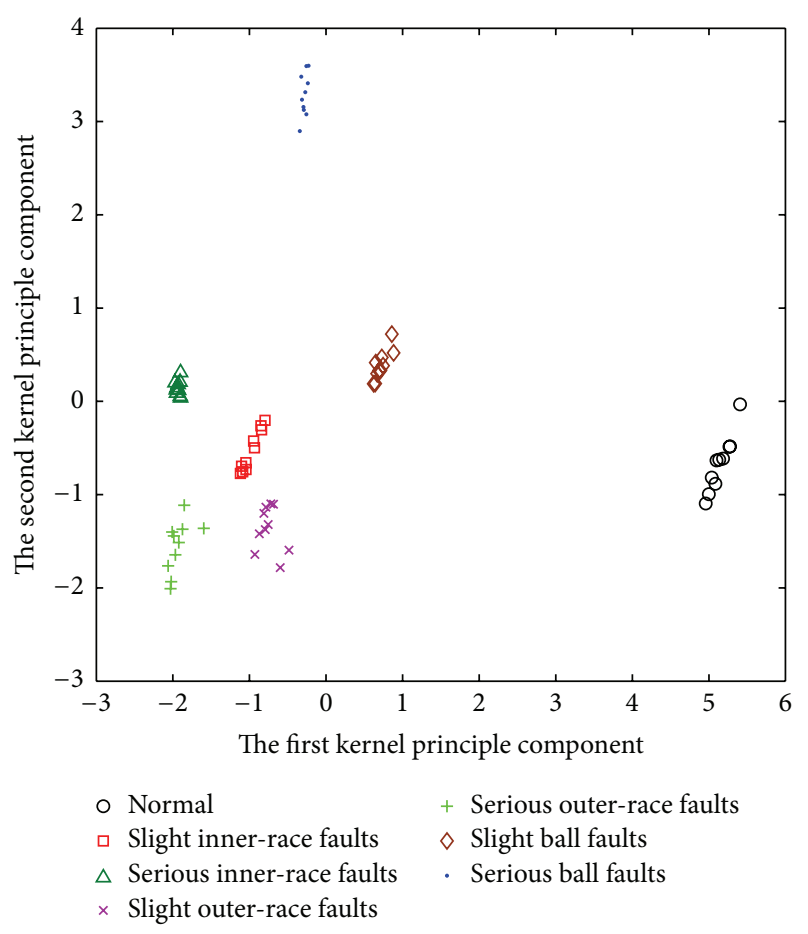

FIGURE 8: The result of classification of seven vibration signals.

published in the recent two years that use the same real data from CWRU is detailed in Table 6. Rauber et al. in [6] used various feature models which are based on the timedomain and frequency-domain parameters, complex envelope spectrum, and wavelet packet analysis, utilized SVM, the $k$-nearest neighbor classifier $(k$-NN), and multilayer perceptron (MLP) for classification of seven failures (normal, two different severities of ball faults, two different severities of inner-race faults, and two different severities of outerrace faults), and reported an accuracy of $98.13 \%, 99.96 \%$, and $99.97 \%$, respectively. Saidi et al. in [18] used the higher order statistics analysis (HOSA) features with feature reduction using PCA and classified the rolling bearing faults into four classes (normal, ball faults, inner-race faults, and outer-race faults) with an accuracy of $96.98 \%$. Zhu et al. in [19] used multiscale entropy (MSE) and hierarchical entropy (HE) and classified ten faults into normal, two different severities of ball faults, four different severities of inner-race faults, and three different severities of outer-race faults, using the PSO-SVM method with an accuracy of $97.75 \%$ and $100 \%$, respectively.

All in all, evaluation of our method versus previous works given in Table 6 shows that our experiment demonstrates the fusion features of MFDFA and ASD, including sufficient fault information of the vibration signals, and suggests that fusion features have good potential for improving diagnostic accuracy of rolling bearings. The fusion feature with the PSOLSSVM classifier produces a good classification performance: the average diagnostic result is $98.6 \%$ in small samples, and the results could be improved if we adopt more data. The method proposed in this paper is promising for a more excellent diagnosis of rolling bearings.
TABLE 5: The diagnosis results comparison of five methods.

\begin{tabular}{lcccccccc}
\hline \multirow{2}{*}{ Feature type } & \multicolumn{8}{c}{ Diagnostic accuracy (\%) } \\
& $S 1$ & $S 2$ & $S 3$ & $S 4$ & $S 5$ & $S 6$ & $S 7$ & Average \\
\hline MFDFA features & 100 & 80 & 100 & 90 & 70 & 90 & 70 & 85.7 \\
ASD features & 80 & 100 & 100 & 80 & 90 & 70 & 100 & 88.6 \\
MFDFA + KPCA & 100 & 100 & 100 & 90 & 70 & 100 & 90 & 92.9 \\
ASD + KPCA & 90 & 100 & 100 & 90 & 100 & 80 & 100 & 92.9 \\
$\begin{array}{l}\text { MFDFA + ASD + } \\
\text { KPCA }\end{array}$ & 100 & 100 & 100 & 90 & 100 & 100 & 100 & 98.6 \\
\hline
\end{tabular}

\section{Conclusions and Future Works}

When rolling bearings fail, the weak fault features are usually buried and make it fairly difficult to determine the damage degree. To solve the problem, this paper introduces a new fault diagnosis method to achieve feature extraction and intelligent classification of different fault position and damage degree of rolling bearing signals based on feature fusion of MFDFA and ASD. Based on the analysis of different operational status data, the following conclusions can be drawn:

(1) By performing MFDFA on the vibration signals of rolling bearing in seven different statuses, the results showed that the values of the five MFDFA features are different from each other and hence can be used as the fault features of rolling bearings.

(2) By fitting ASD to the vibration signals of rolling bearing in seven different statuses and calculating the PDFs, the results showed that the values of the five ASD features are different from each other and can be used as the fault features of rolling bearings too.

(3) By fusing the MFDFA features and ASD features by KPCA, heterogeneous information can complement each other. More abundant information can be obtained while recognition ability of the classifier is improved. The feature fusion method based on MFDFA and ASD can not only achieve the intelligent diagnosis of rolling bearings' fault position and damage degree, but also have better diagnosis accuracy than single feature method and the corresponding single feature fusion method.

There are two possible directions for future works. First, this work has classified the faults of rolling bearings into seven classes under the condition of small samples and should complement more data sets (more fault signals, more training and test samples) to further test its capacity for multiclassification. Secondly, the bearings data from CWRU is relatively clean, and this approach should be applied to 
TABLE 6: Comparison of this work with some previous researches using CWRU bearings data set.

\begin{tabular}{|c|c|c|c|c|c|}
\hline Reference & Features & Classifier & Defects considered & $\begin{array}{c}\text { Classified } \\
\text { states }\end{array}$ & Accuracy (\%) \\
\hline$[4]$ & $\begin{array}{l}\text { Permutation entropy of } \\
\text { intrinsic mode functions } \\
\text { (IMFs) decomposed by } \\
\text { ensemble empirical mode } \\
\text { decomposition (EEMD) }\end{array}$ & $\begin{array}{l}\text { SVM + parameter } \\
\text { optimized by intercluster } \\
\text { distance (ICD) }\end{array}$ & $\begin{array}{l}\mathrm{N}, \mathrm{B}(0.18,0.36,0.54, \\
\text { and } 0.72 \mathrm{~mm}), \mathrm{IR}(0.18, \\
0.36,0.54 \text {, and } 0.72 \mathrm{~mm}), \\
\text { and } \mathrm{OR}(0.18,0.36 \text {, and } \\
0.54 \mathrm{~mm})\end{array}$ & $2,3,4,11$ & $97.91-100$ \\
\hline$[6]$ & $\begin{array}{l}\text { Statistical features from the } \\
\text { time and frequency domains, } \\
\text { wavelet packet energy, and } \\
\text { complex envelope magnitudes }\end{array}$ & $\begin{array}{l}\text { SVM, the } k \text {-nearest } \\
\text { neighbor classifier }(k \text {-NN), } \\
\text { and multilayer perceptron } \\
\text { (MLP) }\end{array}$ & $\begin{array}{l}\mathrm{N}, \mathrm{B}(0.18,0.54 \mathrm{~mm}), \mathrm{IR} \\
(0.18,0.54 \mathrm{~mm}) \text {, and OR } \\
(0.18,0.54 \mathrm{~mm})\end{array}$ & 7 & $98.13-99.97$ \\
\hline [7] & $\begin{array}{l}\text { Skewness, kurtosis, standard } \\
\text { deviation, root mean square } \\
\text { (RMS), crest factor, and five } \\
\text { entropies }\end{array}$ & $\begin{array}{l}\text { SVM and artificial neural } \\
\text { network (ANN) with } \\
\text { different attribute filters }\end{array}$ & $\begin{array}{l}\text { N, B }(0.18,0.54 \mathrm{~mm}), \text { IR } \\
(0.18,0.54 \mathrm{~mm}) \text {, and OR } \\
(0.18,0.54 \mathrm{~mm})\end{array}$ & 7 & 100 \\
\hline [18] & $\begin{array}{l}\text { Features derived from higher } \\
\text { order statistics analysis } \\
\text { (HOSA) with feature } \\
\text { reduction using PCA }\end{array}$ & $\begin{array}{l}\text { SVM + one against all } \\
\text { (OAA) }\end{array}$ & $\begin{array}{l}\mathrm{N}, \mathrm{B}(0.18,0.36,0.54, \\
\text { and } 0.72 \mathrm{~mm}), \mathrm{IR}(0.18, \\
0.36,0.54 \text {, and } 0.72 \mathrm{~mm}), \\
\text { and } \mathrm{OR}(0.18,0.36 \text {, and } \\
0.54 \mathrm{~mm})\end{array}$ & 4 & 96.98 \\
\hline [19] & $\begin{array}{l}\text { Multiscale entropy (MSE), } \\
\text { hierarchical entropy (HE) }\end{array}$ & $\begin{array}{l}\text { SVM + parameter } \\
\text { optimized by PSO }\end{array}$ & $\begin{array}{l}\mathrm{N}, \mathrm{B}(0.18,0.72 \mathrm{~mm}), \mathrm{IR} \\
(0.18,0.36,0.54 \text {, and } \\
0.72 \mathrm{~mm}), \text { and } \mathrm{OR}(0.18, \\
0.36 \text {, and } 0.54 \mathrm{~mm})\end{array}$ & 10 & $\begin{array}{c}\text { MSE + SVM: } 97.75 \\
\text { HE + SVM: } 100\end{array}$ \\
\hline$[20]$ & $\begin{array}{l}\text { Statistical features from the } \\
\text { time and frequency domains, } \\
\text { the energy of empirical mode } \\
\text { decomposition (EMD) }\end{array}$ & $\begin{array}{l}\text { Statistical locally linear } \\
\text { embedding (S-LLE) + } \\
k \text {-NN, classification and } \\
\text { regression trees (CART), } \\
\text { and RBF-SVM }\end{array}$ & $\begin{array}{l}\mathrm{N}, \mathrm{B}(0.54 \mathrm{~mm}), \mathrm{IR} \\
(0.54 \mathrm{~mm}), \text { and OR } \\
(0.54 \mathrm{~mm})\end{array}$ & 4 & 97.26 \\
\hline [21] & $\begin{array}{l}\text { Features extracted from } \\
\text { wavelet kurtogram and } \\
\text { quefrency cepstrum }\end{array}$ & $\begin{array}{l}\text { Swarm Rapid Centroid } \\
\text { Estimation (SRCE) + } \\
\text { Hidden Markov Model } \\
\text { (HMM) }\end{array}$ & $\begin{array}{l}\mathrm{N}, \mathrm{B}(0.54 \mathrm{~mm}), \mathrm{IR} \\
(0.54 \mathrm{~mm}) \text {, and OR } \\
(0.54 \mathrm{~mm} \text { located at } \\
\text { three different positions })\end{array}$ & $\begin{array}{l}\text { Drive end: } 5 \\
\text { Fan end: } 5\end{array}$ & $\begin{array}{l}\text { Drive end: } 95.08 \\
\text { Fan end: } 100\end{array}$ \\
\hline Present work & $\begin{array}{l}\text { Features derived from } \\
\text { MFDFA and ASD with feature } \\
\text { fusion using KPCA }\end{array}$ & $\begin{array}{l}\text { LSSVM + parameter } \\
\text { optimized by PSO }\end{array}$ & $\begin{array}{l}\mathrm{N}, \mathrm{B}(0.18,0.54 \mathrm{~mm}), \mathrm{IR} \\
(0.18,0.54 \mathrm{~mm}), \text { and OR } \\
(0.18,0.54 \mathrm{~mm})\end{array}$ & 7 & 98.6 \\
\hline
\end{tabular}

$\mathrm{N}$ denotes normal, $\mathrm{B}$ denotes ball faults, IR denotes inner-race faults, and OR denotes outer-race faults.

detect the various fault signals of rolling bearings in actual projects which contain a lot of noise.

\section{Conflict of Interests}

The authors declare that there is no conflict of interests regarding the publication of this paper.

\section{Acknowledgments}

The authors would like to sincerely thank Bearing Data Center of Case Western Reserve University for providing bearing fault data. The work was supported by Joint Fund of National Basic Research of High Speed Railway (no. U1234208); Science and Technology Support Program (no. CE20130041); Independent Research Project of National Key Laboratory (no. 2013TPL_T04); and Sichuan Province Based Application Program (no. 2014JY0078).

\section{References}

[1] Z. Zhang, X. Shi, Q. Shi, and B. Tang, "Fault feature extraction of rolling element bearing based on improved EMD and spectral kurtosis," Journal of Vibration, Measurement and Diagnosis, vol. 33, no. 3, pp. 478-482, 2013.

[2] A. Bourdon, D. Rémond, S. Chesné, and H. André, "Reconstruction of the instantaneous angular speed variations caused by a spall defect on a rolling bearing outer ring correlated with the length of the defect," in Advances in Condition Monitoring of Machinery in Non-Stationary Operations, Lecture Notes in Mechanical Engineering, pp. 335-345, Springer, Berlin, Germany, 2014.

[3] J. B. Ali, N. Fnaiech, L. Saidi, B. Chebel-Morello, and F. Fnaiech, "Application of empirical mode decomposition and artificial neural network for automatic bearing fault diagnosis based on vibration signals," Applied Acoustics, vol. 89, pp. 16-27, 2015.

[4] X. Zhang, Y. Liang, J. Zhou, and Y. zang, "A novel bearing fault diagnosis model integrated permutation entropy, ensemble 
empirical mode decomposition and optimized SVM," Measurement, vol. 69, pp. 164-179, 2015.

[5] M. Kang, J. Kim, and J.-M. Kim, "Reliable fault diagnosis for incipient low-speed bearings using fault feature analysis based on a binary bat algorithm," Information Sciences, vol. 294, pp. 423-438, 2015.

[6] T. W. Rauber, F. de Assis Boldt, and F. M. Varejão, "Heterogeneous feature models and feature selection applied to bearing fault diagnosis," IEEE Transactions on Industrial Electronics, vol. 62, no. 1, pp. 637-646, 2015.

[7] A. Sharma, M. Amarnath, and P. Kankar, "Feature extraction and fault severity classification in ball bearings," Journal of Vibration and Control, 2014.

[8] Z. Wu and N. E. Huang, "Ensemble empirical mode decomposition: a noise-assisted data analysis method," Advances in Adaptive Data Analysis, vol. 1, no. 1, pp. 1-41, 2009.

[9] H. Liu and M. Han, "A fault diagnosis method based on local mean decomposition and multi-scale entropy for roller bearings," Mechanism and Machine Theory, vol. 75, pp. 67-78, 2014.

[10] P. Li, Y. Jiang, and J. Xiang, "Experimental investigation for fault diagnosis based on a hybrid approach using wavelet packet and support vector classification," The Scientific World Journal, vol. 2014, Article ID 145807, 10 pages, 2014.

[11] E. A. F. Ihlen and B. Vereijken, "Multifractal formalisms of human behavior," Human Movement Science, vol. 32, no. 4, pp. 633-651, 2013.

[12] E. A. F. Ihlen, "The influence of power law distributions on longrange trial dependency of response times," Journal of Mathematical Psychology, vol. 57, no. 5, pp. 215-224, 2013.

[13] W.-C. Yang, C.-H. Zhao, and B.-Z. Cheng, "Recognition of communication signals in noise with alpha stable distribution," Journal of Applied Sciences, vol. 28, no. 2, pp. 111-114, 2010.

[14] H. Liu, X. Wang, and C. Lu, "Rolling bearing fault diagnosis based on LCD-TEO and multifractal detrended fluctuation analysis," Mechanical Systems and Signal Processing, vol. 60-61, pp. 273-288, 2015.

[15] J. Lin and Q. Chen, "Fault diagnosis of rolling bearings based on multifractal detrended fluctuation analysis and Mahalanobis distance criterion," Mechanical Systems and Signal Processing, vol. 38, no. 2, pp. 515-533, 2013.

[16] G. Yu, C. Li, and J. Zhang, "A new statistical modeling and detection method for rolling element bearing faults based on alphastable distribution," Mechanical Systems and Signal Processing, vol. 41, no. 1-2, pp. 155-175, 2013.

[17] G. Yu and N. Shi, "Gear fault signal modeling and detection based on alpha stable distribution," in Proceedings of the International Symposium on Instrumentation and Measurement, Sensor Network and Automation (IMSNA '12), pp. 471-474, Sanya, China, August 2012.

[18] L. Saidi, J. Ben Ali, and F. Fnaiech, "Application of higher order spectral features and support vector machines for bearing faults classification," ISA Transactions, vol. 54, pp. 193-206, 2015.

[19] K. Zhu, X. Song, and D. Xue, "A roller bearing fault diagnosis method based on hierarchical entropy and support vector machine with particle swarm optimization algorithm," Measurement, vol. 47, no. 1, pp. 669-675, 2014.

[20] X. Wang, Y. Zheng, Z. Zhao, and J. Wang, "Bearing fault diagnosis based on statistical locally linear embedding," Sensors, vol. 15, no. 7, pp. 16225-16247, 2015.
[21] M. Yuwono, Y. Qin, J. Zhou, Y. Guo, B. G. Celler, and S. W. Su, "Automatic bearing fault diagnosis using particle swarm clustering and Hidden Markov Model," Engineering Applications of Artificial Intelligence, 2015.

[22] J. W. Kantelhardt, S. A. Zschiegner, E. Koscielny-Bunde, S. Havlin, A. Bunde, and H. E. Stanley, "Multifractal detrended fluctuation analysis of nonstationary time series," Physica A: Statistical Mechanics and Its Applications, vol. 316, no. 1-4, pp. 87-114, 2002.

[23] P. Shang, Y. Lu, and S. Kamae, "Detecting long-range correlations of traffic time series with multifractal detrended fluctuation analysis," Chaos, Solitons and Fractals, vol. 36, no. 1, pp. 82-90, 2008.

[24] G. Samorodnitsky and M. S. Taqqu, Stable Non-Gaussian Random Processes: Stochastic Models with Infinite Variance, Chapman \& Hall, Boca Raton, Fla, USA, 1994.

[25] M. Shao and C. L. Nikias, "Signal processing with fractional lower order moments: stable processes and their applications," Proceedings of the IEEE, vol. 81, no. 7, pp. 986-1010, 1993.

[26] B. Schölkopf, A. Smola, and K.-R. Müller, "Nonlinear component analysis as a Kernel Eigenvalue Problem," Neural Computation, vol. 10, no. 5, pp. 1299-1319, 1998.

[27] M. Žvokelj, S. Zupan, and I. Prebil, "Non-linear multivariate and multiscale monitoring and signal denoising strategy using Kernel Principal Component Analysis combined with Ensemble Empirical Mode Decomposition method," Mechanical Systems and Signal Processing, vol. 25, no. 7, pp. 2631-2653, 2011.

[28] Y. del Valle, G. K. Venayagamoorthy, S. Mohagheghi, J.-C. Hernandez, and R. G. Harley, "Particle swarm optimization: basic concepts, variants and applications in power systems," IEEE Transactions on Evolutionary Computation, vol. 12, no. 2, pp. 171-195, 2008.

[29] J. A. K. Suykens and J. Vandewalle, "Least squares support vector machine classifiers," Neural Processing Letters, vol. 9, no. 3, pp. 293-300, 1999.

[30] Z. Yang, T. Peng, J. Li, and Y. Zhong, "Bayesian inference LSSVM based fault diagnosis method for rolling bearing," Journal of Electronic Measurement and Instrument, vol. 24, no. 5, pp. 420-424, 2010.

[31] K. A. Loparo, Bearings Vibration Data Set, [EB/OL], Case Western Reserve University, 2008, http://csegroups.case.edu/bearingdatacenter/pages/download-data-file.

[32] I. A. Koutrouvelis, "An iterative procedure for the estimation of the parameters of stable laws," Communications in Statistics. B. Simulation and Computation, vol. 10, no. 1, pp. 17-28, 1981. 


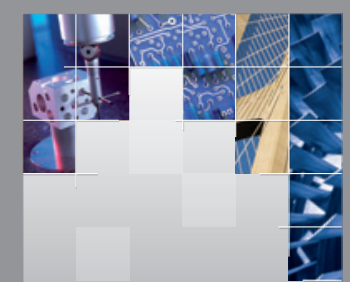

\section{Enfincering}
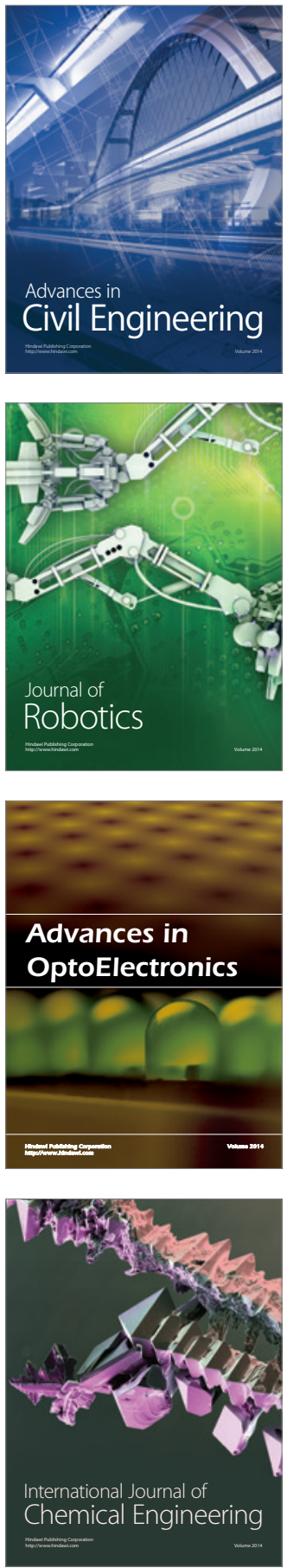

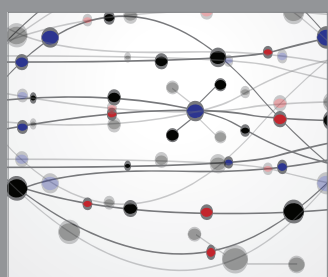

The Scientific World Journal

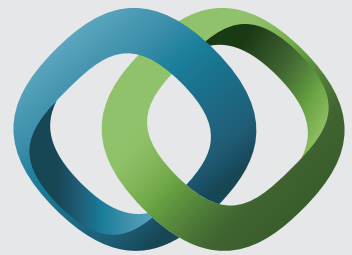

\section{Hindawi}

Submit your manuscripts at

http://www.hindawi.com
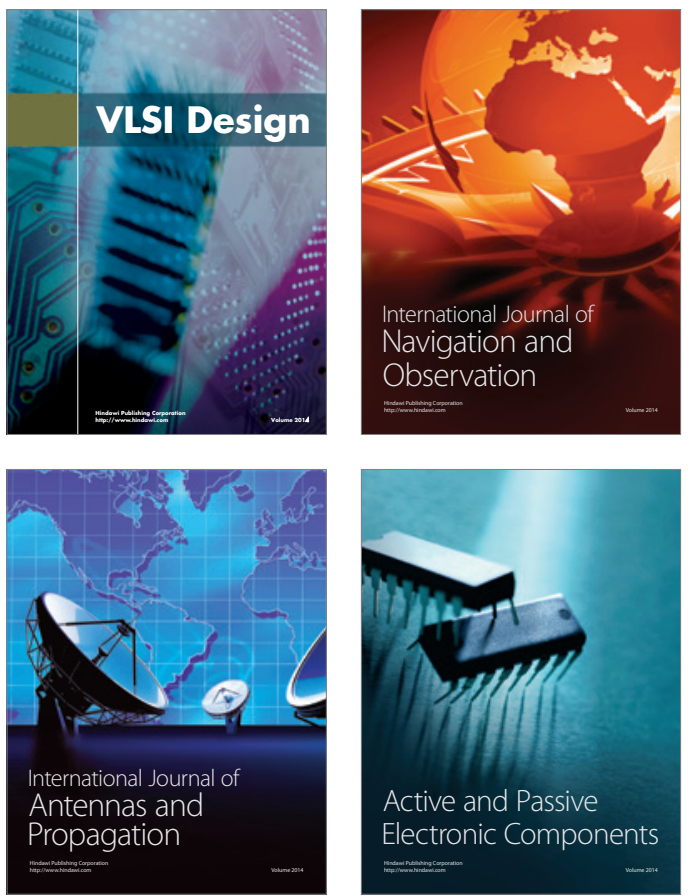
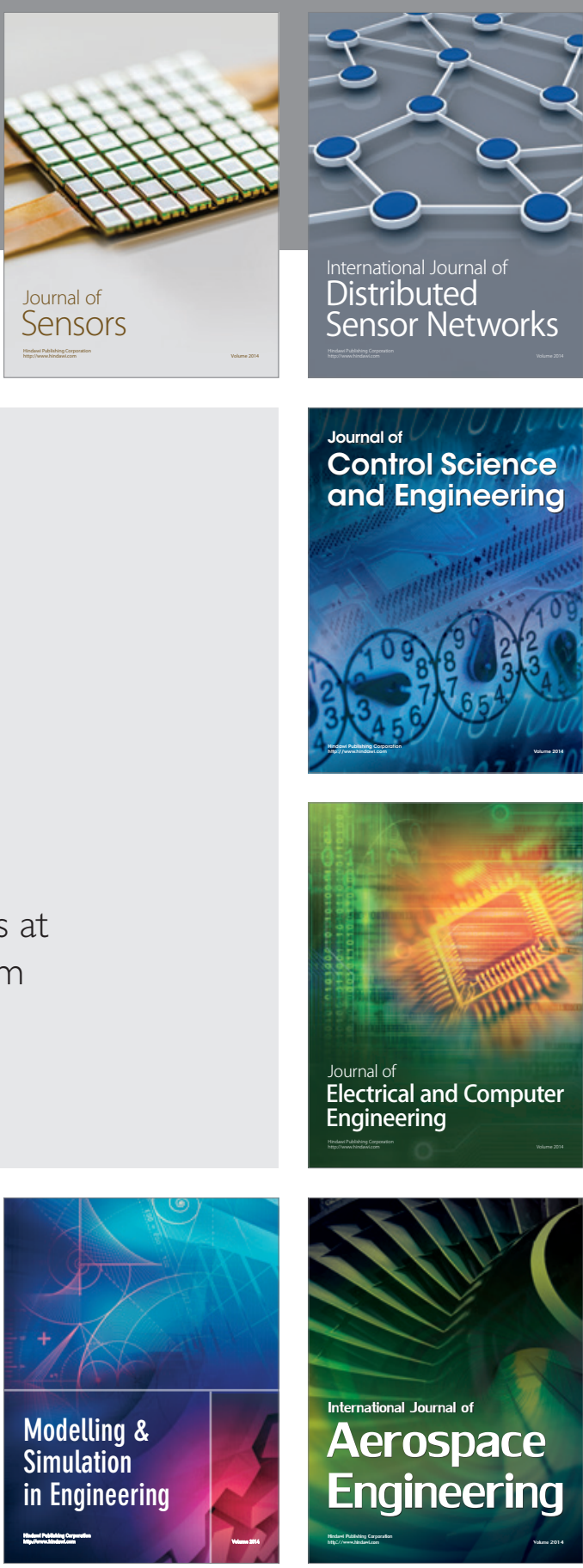

International Journal of

Distributed

Sensor Networks

Journal of

Control Science

and Engineering
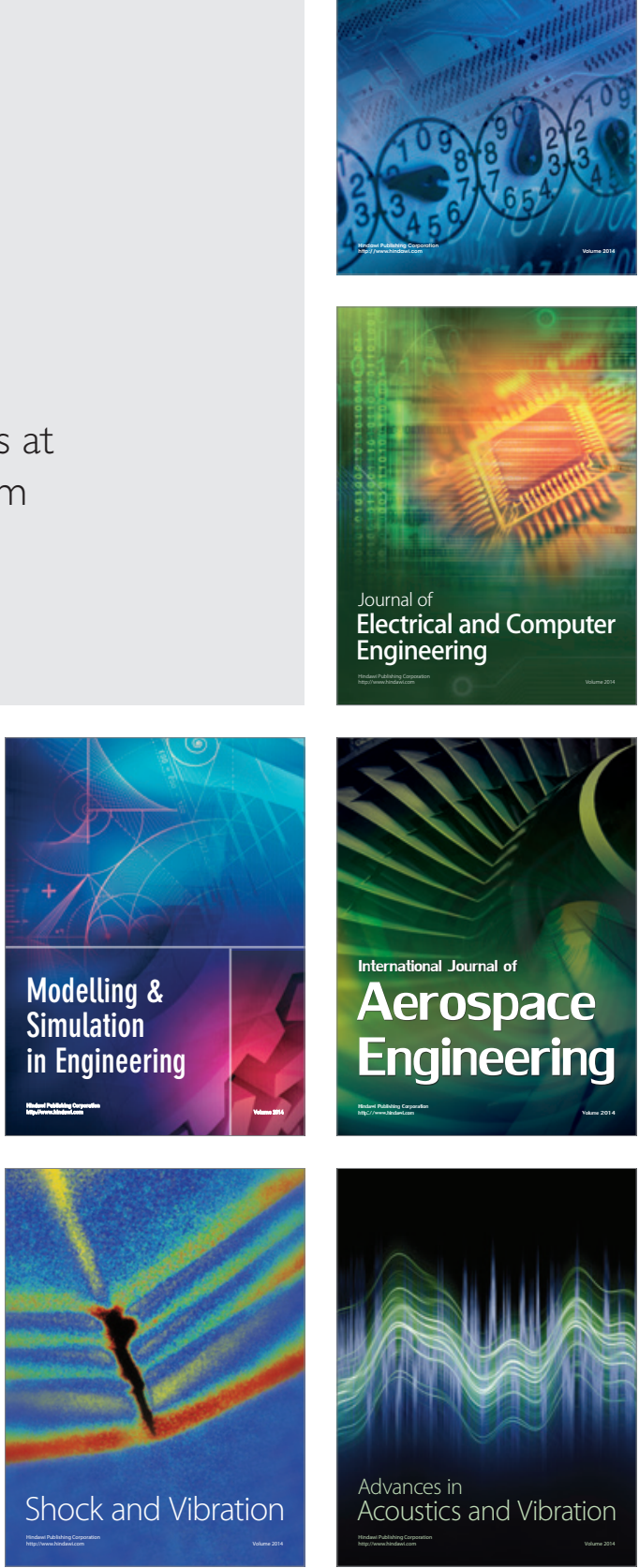